\title{
Characterization of a spherical heat source for measuring thermal conductivity and water content of ethanol and water mixtures
}

\author{
Júlio Dutra Brionizio, ${ }^{1, *}$, Alcir de Faro Orlando ${ }^{2}$, and Georges Bonnier ${ }^{3}$ \\ ${ }^{1}$ National Institute of Metrology, Quality and Technology (Inmetro), Av. Nossa Senhora das Graças, 50, Xerém, \\ Duque de Caxias, RJ, CEP: 25250-020, Brazil \\ 2 Pontifical Catholic University of Rio de Janeiro (PUC-Rio), Rua Marquês de São Vicente, 225, Gávea, Rio de Janeiro, \\ RJ, CEP: 22451-900, Brazil \\ ${ }^{3}$ Georges Bonnier Consultant, Rue Charles Perrault, 14, Montsoult 95560, France
}

Received: 4 July 2016 / Accepted: 2 May 2017

\begin{abstract}
The study and the development of measuring methods of thermal conductivity are essential in several engineering applications, since as a consequence of the current justified demands on saving and rational use of thermal energy, the heat transfer with the maximum efficient as possible is of great relevance. The measurement of the water content is also a relevant parameter in several research areas and industrial sectors, since the quantity of water in the substances influences several biological, chemical and physical processes. The aim of this paper is to present an experimental and theoretical study, following the good metrological practices, of a method based on a spherical heat source in order to measure the thermal conductivity of liquids, focusing on water, ethanol and their mixtures, with later determination of the water content of the binary samples.
\end{abstract}

Keywords: thermal conductivity / water content / spherical heat source / metrology

\section{Introduction}

Due to the environmental needs, the rational use and saving of thermal energy have become increasingly relevant in the last decades, especially in the industrial sectors. Thus, the heat transfer with the best efficiency is as important as minimizing the heat loss through the use of appropriate materials. Hence, the knowledge of the thermal conductivity of materials is of great relevance in several engineering applications where heat transfer plays a fundamental role. The water content is also another important quantity for many industrial sectors and research areas, since the amount of water in the substances influences several biological, chemical and physical processes. Nevertheless, the number of equipment available on the market for the measurement of these quantities is not vast when compared to other quantities, mainly for liquid substances. For this reason, researches on the development of alternative measuring techniques have considerable significance.

The thermal conductivity is a transport property that furnishes an indication of the rate at which energy is transferred by the conduction process (net transfer of

* Corresponding author: jdbrionizio@inmetro.gov.br energy by random molecular motion). The measuring methods of the thermal conductivity are often classified as being steady-state and transient. In the case of fluids, its measurement is more difficult than that of solids due to the possibility of the onset of natural convection, which consists in energy transfer due to random molecular motion and the global motion of the fluid. This fluid motion is due to the buoyancy forces induced by density differences caused by temperature variations in the fluid.

The thermal conductivity depends on several parameters, and the water content is one of them. Consequently, the water content can be indirectly determined by means of the thermal conductivity value of the material, which is higher as the water content increases, since the thermal conductivity of water is higher than that of many nonmetallic solids, gases and liquids.

For a long while researchers have been working on new approaches for theoretical estimation of thermal conductivity of liquids, by linking it to other physical property, usually using temperature as a variable, or by deriving a model from the existing ones. Also, several experiments have been developed, using different techniques based on steady-state and transient methods, in order to measure it properly.

The line heat source probe (hot-wire method) is usually recognized as the most accurate technique for measuring thermal conductivity of liquids. The concept and the initial 
experiments with heated wires started about 1780, and the first transient hot-wire instrument was proposed by Stâlhane and Pyk in 1931 to measure the thermal conductivity of solids, powders and some liquids. Since 1780, the method has been studied by several researchers [1]. The standard test method for the determination of thermal conductivity of nonmetallic liquids of the American Society for Testing and Materials (ASTM) is based on this method [2]. An extensive uncertainty assessment elaborated for the hot-wire method obtained the value of $5.8 \%$ for the thermal conductivity [3], although other literatures claim for uncertainty values better than $5 \%$.

Models based on spherical heat sources have also been studied to measure thermal conductivity of different materials (solid, powder, paste, etc.). In this technique, the spherical device simultaneously generates heat and monitors the temperature response with time. Heat sources of spherical symmetry are free of lateral thermal effects and they yield to the steady-state regime at long times.

In 1933, Gibbs [4] described a device primarily designed to determine qualitatively the changes in blood flow through a tissue or organ by means of an electrically heated thermocouple. From 1951, Grayson [5-7] discussed the use of the apparatus for measuring the thermal conductivity, taking into consideration a steady-state regime in which the relation between heat production and heat loss is represented by equation (2), described by Carslaw [8] in 1921 for an electrically heated spherical source in an infinite mass of material. In 1968, Chato [9] was to first to use a thermistor as temperature sensor and heating element for measuring thermal conductivity and thermal diffusivity of biological materials. Several other researchers have also applied the thermistor method for measuring thermal properties of biomaterials [10-15]. Woodbury [16] used the thermistor method to measure thermal conductivity of building insulation with varying degree of wetness. Fujii et al. [17] employed the technique to measure thermal conductivities of liquid mixtures of water-ethanol, water-methanol and R113-oil. Dougherty [18] used the thermistor method to perform thermal conductivity measurements in materials ranging from low viscosity fluids to insulation ones. Kravets [19] used the technique to measure thermal conductivity of milk and cream over the range of $25^{\circ} \mathrm{C}$ to $125^{\circ} \mathrm{C}$. Holeschovsky et al. [20] used the thermistor method to measure the thermal conductivity of liquids and silica gel. Radhakrishnan [21] measured the thermal properties of ten different seafood in the range from $5{ }^{\circ} \mathrm{C}$ to $30^{\circ} \mathrm{C}$. Gelder [22] employed the thermistor technique to measure thermal properties of moist food materials at high temperatures. In 2003, Zhang et al. [23], based on a transient thermal model of a point heat pulse, developed a dual-thermistor probe, in which two thermistor beads serve as point heater and temperature sensor, in order to measure thermal diffusivity and thermal conductivity. From 2005, Kubicar et al. [24-26] presented a sensor to measure thermal conductivity, in which a heat source and a thermometer were unified in a single component in the form of a small ball.
In principle, a model based on a spherical heat source is an absolute measuring method of thermal conductivity, which means that the sensor can furnish a result without being calibrated against a standard or a reference material. Nevertheless, parameters of the model need to be determined or obtained by means of calibration.

The research was focused on ethanol because it is a strategic biofuel in Brazil, and also because it is widely used as feedstock (pure or mixed with water) in several industrial sectors, such as pharmaceutical, food processing, hygiene and others. Since the early days, ethanol has helped fuel motorized transport in Brazil. In 1903, Brazil's First National Congress on Industrial Applications of Alcohol recommended the development of infrastructure to produce automotive ethanol. Several ethanol programs were implemented by the Brazilian government since then [27]. As a consequence, many ethanol production plants are spread all over the country, which makes Brazil the world's second largest producer of ethanol fuel. So, studies on ethanol are of great relevance for the industries, especially those related to measurement processes.

\section{Theoretical model}

In the measuring principle of the thermal properties by spherical geometry, the sensor is inserted in the medium of interest. Electrical power is supplied to the sensor and a constant heat flow is generated inside. Through the radius of the sphere, the heat propagates through the surrounding medium by a radial distance, resulting in temperature rise of the medium until its stabilization. The heat dissipated by the ball to maintain this temperature rise depends on the thermal properties of the medium.

The heat transfer model of a spherical heat source is generally based on the following hypothesis: the sensor and the medium are in thermal equilibrium before heating; the surrounding medium is homogeneous, isotropic and infinite; the sensor is homogeneous and isotropic; the sensor has spherical shape; the heat is generated uniformly within the sensor; there is no thermal contact resistance between the sensor and the environment; and the only heat transfer mode acting in the process is conduction.

The temperature rise of the spherical heat source and the measured medium in the steady-state regime can be described by the following initial and boundary conditions:

$$
\begin{array}{ll}
T_{s}=T_{m}=0 & \text { when } t=0 \\
T_{m} \rightarrow 0 & \text { when } r \rightarrow \infty \text { and } t>0 \\
T_{s}=T_{m} & \text { when } r=r_{s} \text { and } t>0 \\
\partial \frac{T_{s}}{\partial r}=0 & \text { when } r=0 \text { and } t>0 \\
k_{s} \frac{\partial T_{s}}{\partial r}=k_{m} \frac{\partial T_{m}}{\partial r} & \text { when } r=r_{s} \text { and } t>0
\end{array}
$$

where the subscripts $s$ and $m$ represent the sphere and the medium, respectively; $T$ is the temperature; $t$ is the time; $r$ is the radial distance; $r_{s}$ is the radius of the sphere; and $k$ is the thermal conductivity. 

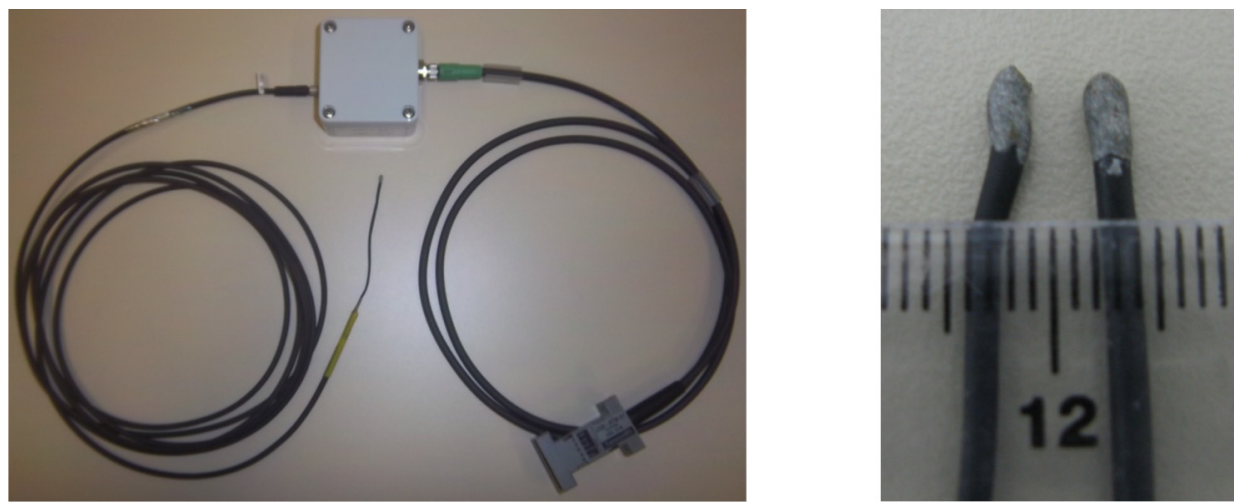

Fig. 1. Measuring system (left) and spherical sensors (right).

The temperature distribution of a region bounded internally by a sphere, with initial temperature as zero and constant heat flux in the interface, is given by [28]:

$$
\begin{aligned}
& T_{m}=\frac{q^{\prime \prime} r_{s}^{2}}{k_{m} r}\left\{\operatorname{erfc} \frac{r-r_{s}}{2 \sqrt{\alpha_{m} t}}\right. \\
&\left.-\exp \left[\frac{r-r_{s}}{r_{s}}+\frac{\alpha_{m} t}{r_{s}^{2}}\right] \operatorname{erfc}\left[\frac{r-r_{s}}{2 \sqrt{\alpha_{m} t}}+\frac{\sqrt{\alpha_{m} t}}{r_{s}}\right]\right\},
\end{aligned}
$$

where $q$ 'l is the heat transfer per unit area (heat flux) and $\operatorname{erfc}(x)$ is the complementary error function.

For a temperature measured at the surface of the sphere $\left(r=r_{s}\right)$ at long times $(t \rightarrow \infty)$, an equation for determining the thermal conductivity of the medium that surrounds the sensor in the steady-state regime can be obtained, as previously shown by Carslaw [8]:

$$
k_{m}=\frac{q^{\prime \prime} r_{s}}{T_{m}}=\frac{\dot{Q}}{4 \pi r_{s} T_{m}},
$$

where $\dot{Q}$ is the heat transfer rate.

The temperature distribution within a sphere, with initial temperature as zero, considering its heat capacity and the thermal contact resistance between the heated sphere and the surrounding medium is given by [28]:

$$
\begin{aligned}
T_{s} & =\frac{\dot{Q}}{4 \pi r_{s} k_{m}}\left[\frac{1+r_{s} \phi}{r_{s} \phi}\right. \\
& \left.-\frac{2 r_{s}^{2} \gamma^{2} \phi^{2}}{\pi} \int_{0}^{\infty} \frac{\exp \left(-\alpha_{m} u^{2} t / r_{s}^{2}\right)}{\left[u^{2}\left(1+r_{s} \phi\right)-\gamma r_{s} \dot{\phi}^{2}\right]+\left(u^{3}-\gamma r_{s} \phi u^{2}\right)} d u\right],
\end{aligned}
$$

where $\gamma=4 \pi r_{s}^{3} \rho_{m}\left(c_{m} / m_{s} c_{s}\right) ; \phi=H_{c} / k_{m}=1 / k_{m} R_{c} ; \rho$ is the density; $c$ is the specific heat; $m$ is the mass; and $H_{c}$ is the thermal contact conductance, which is the inverse of the thermal contact resistance $\left(R_{c}\right)$.

For the steady-state regime, the temperature distribution within a sphere, with initial temperature as zero, considering the thermal contact resistance between the heated sphere and the surrounding medium is given by [28]:

$$
\begin{aligned}
T_{s} & =\dot{q} \frac{\left[r_{s}^{2}-r^{2}+2 R_{c} r_{s} k_{s}+2 r_{s}^{2}\left(k_{s} / k_{m}\right)\right]}{6 k_{s}} \\
& =\dot{Q} \frac{\left[r_{s}^{2}-r^{2}+\left(2 / H_{c}\right) r_{s} k_{s}+2 r_{s}^{2}\left(k_{s} / k_{m}\right)\right]}{8 \pi k_{s} r_{s}^{3}}
\end{aligned}
$$

where $\dot{q}$ is the heat transfer rate per unit volume.

\section{Materials and experimental methods}

Two commercial measuring systems were used in the experiments. Each instrument is composed by a remote spherical sensor and an electronic unit. The sensor consists of a NTC (Negative Temperature Coefficient) thermistor of $47 \mathrm{k} \Omega$ and an electrical resistance of $100 \Omega$ fixed together by means of epoxy resin, resulting in a sphere with nominal diameter of $2 \mathrm{~mm}$. The operating range of the thermistor is $-40^{\circ} \mathrm{C}$ to $150{ }^{\circ} \mathrm{C}$, the temperature response is $0.1^{\circ} \mathrm{C}$ to $5^{\circ} \mathrm{C}$, and the power generation range of the device is $1 \mathrm{~mW}$ to $30 \mathrm{~mW}$. Figure 1 shows one measuring system (left) and the spherical sensors (right).

The rate of heat transfer from the heated spherical sensor to the medium results from the generating process of internal power, which comes from the conversion of electrical energy into thermal energy. This phenomenon occurs due to resistive heating, i.e., an electric current $(I)$ passes through a resistance $(R)$ inside the sensor (control volume), electric power is then dissipated at a rate equal to $I^{2} R$ which corresponds to the rate at which thermal energy is released.

The thermistors were calibrated in the range from $15^{\circ} \mathrm{C}$ to $30^{\circ} \mathrm{C}$, in steps of $2.5^{\circ} \mathrm{C}$, by comparison against a platinum resistance thermometer of $100 \Omega$ (at $0^{\circ} \mathrm{C}$ ) calibrated by the Thermometry Laboratory of Inmetro. The electrical power values adjusted in the devices were compared to those calculated by means of voltage and current measurements performed by precise digital multimeters (of $6 \frac{1}{2}$ and $7 \frac{1}{2}$ digits of resolution) calibrated by the Voltage and Electric Current Laboratory of Inmetro. The diameters of the sensors were measured by the Dimensional Metrology Laboratory of Inmetro by means of an optical 


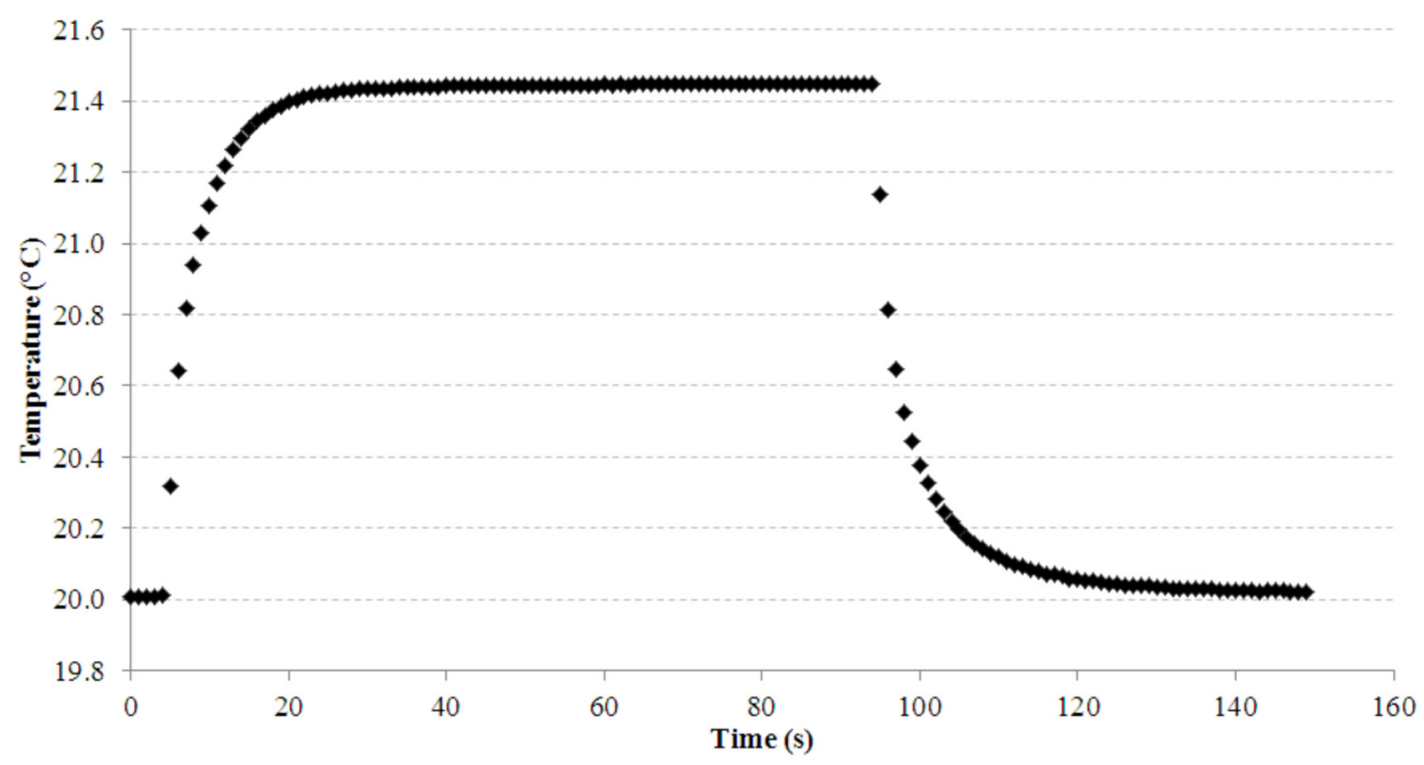

Fig. 2. Temperature monitoring during a measurement cycle.

microscope traceable to a standard ruler, which in turn was calibrated by a He-Ne laser interferometer. The measurement results of the diameter of the sensors were $2.32 \mathrm{~mm}$ (sensor 01) and $2.08 \mathrm{~mm}$ (sensor 02), both having expanded measurement uncertainty of $0.03 \mathrm{~mm}$, with coverage probability of $95.45 \%$.

For the measurements, the following samples were used: distilled water, anhydrous ethanol (purity around $99.3 \%$ ), ethyl alcohol (purity around 95\%), solution of $75 \%$ of ethanol and $25 \%$ of water, solution of $50 \%$ of ethanol and $50 \%$ of water, and solution of $25 \%$ of ethanol and $75 \%$ of water. The water content of the binary samples was measured by the Organic Analysis Laboratory of Inmetro by means of a Karl Fischer coulometric titration. All the samples were stored in dark borosilicate glass bottles with the same characteristics and size. The samples had approximately volume of $1 \mathrm{~L}$, although lower volumes could be used. In previous works $[15,29,30]$, the volume of the sample was estimated from 5 to 12 times the radius of the sensor, resulting in values in the order of $1 \mathrm{~cm}^{3}$.

When measuring the thermal conductivity of fluids, it is desirable that the heat transfer from the measuring system to the medium should occur only by conduction. So, care must be taken to prevent the onset of natural convection. In order to detect its onset, the experimental method developed by Schmidt and Milverton [31] was used. The researchers noted that in the heat transfer by conduction, the temperature difference imposed in the fluid increased linearly with the power supplied to the heating system of the apparatus, and this linearity was interrupted when convection started to act. Therefore, the spherical sensors were immerged in distilled water and ethyl alcohol at $20^{\circ} \mathrm{C}$, and thirty nominal values of electrical power (with increments of approximately $0.45 \mathrm{~mW}$ ) were set in the devices in the range from $1 \mathrm{~mW}$ to $14 \mathrm{~mW}$. For both sensors, it was clearly observed that the relation between the electrical power and the temperature increment was linear for the whole range. Nevertheless, for ethyl alcohol, this linearity was interrupted from $8 \mathrm{~mW}$ and $6 \mathrm{~mW}$ for the sensors numbered as 01 and 02 , respectively, which is an indication of the convection onset.

The spherical sensor of the measuring system was placed approximately in the middle of the bottle, which in turn was conditioned at $20^{\circ} \mathrm{C}$ in a thermostatic bath with stability lower than $0.02^{\circ} \mathrm{C}$. The sample within the bottle was completely covered by the fluid of the bath. The sensor was guided to the middle of the bottle by means of a very small hole prepared in the removable lid of the container. In order to avoid infiltration of air to the inner of the bottle, the tip and part of the sensor cable were wrapped with polyvinyl chloride (PVC) film. After removal of the spherical sensor from a sample, it was exhaustively rinsed with distilled water and dried with paper towel and hot air before submerging it on another sample. This was done to prevent cross-contamination.

Before starting the measurements, the samples were left for at least $12 \mathrm{~h}$ in the bath so as to guarantee their completely stabilization at $20^{\circ} \mathrm{C}$. The nominal electrical powers set in the electronic units were, respectively, $8 \mathrm{~mW}$ and $6 \mathrm{~mW}$ for the spherical sensors 01 and 02 . Several measurement cycles were performed for each sensor, where each one was composed by temperature monitoring before, during and after the heating of the spherical sensor. Five measurements were acquired before the heating of the sphere, ninety-five measurements were obtained during it, and fifty measurements were collected when the heating process was stopped. The interval between measurements was $1 \mathrm{~s}$, so that each cycle took $2.5 \mathrm{~min}$ from the beginning to the end. An interval from $10 \mathrm{~min}$ to $20 \mathrm{~min}$ between cycles was given so as to prevent that the temperature rise of a cycle could affect the following one. This was confirmed by comparing the first measurement of a cycle with the last one of the previous cycle.

The thermal conductivity of the samples was determined in the steady-state regime. For this reason, it was very important to establish when this regime was reached. During the calibration of the thermometers, where several 
Table 1. Concentrations and expanded uncertainties of the samples.

\begin{tabular}{lccl}
\hline$E C_{N}(\%$ ethanol $)$ & $W C_{N}(\%$ water $)$ & $W C_{R}(\%$ water $)$ & $U\left(W C_{R}\right)(\%$ water $)$ \\
\hline 99.3 & 0.7 & 0.720 & 0.004 \\
95 & 5 & 5.368 & 0.001 \\
75 & 25 & 25.5270 & 0.2143 \\
50 & 50 & 50.3596 & 0.2862 \\
25 & 75 & 75.1930 & 0.1509 \\
\hline
\end{tabular}

Table 2. Coefficients $\phi$ of the equation of Assael et al. [32].

\begin{tabular}{lcc}
\hline & $\phi_{1}(\mathrm{~mW} / \mathrm{m} \mathrm{K})$ & $\phi_{2}(\mu \mathrm{W} / \mathrm{m} \mathrm{K})$ \\
\hline Water & 209.2 & 1342 \\
$100 \%$ methanol & 220.7 & -75 \\
$75 \%$ methanol $+25 \%$ water & 185.5 & 249 \\
$50 \%$ methanol $+50 \%$ water & 147.6 & 639 \\
$25 \%$ methanol $+75 \%$ water & 73.7 & 1247 \\
$100 \%$ ethanol & 186.4 & -84 \\
$75 \%$ ethanol $+25 \%$ water & 198.7 & 95 \\
$50 \%$ ethanol $+50 \%$ water & 178.5 & 443 \\
$25 \%$ ethanol $+75 \%$ water & 75.8 & 1190 \\
$100 \%$ propanol & 179.2 & -100 \\
$50 \%$ propanol $+50 \%$ water & 139.2 & 565 \\
\hline
\end{tabular}

measurements were taken during $30 \mathrm{~min}$ after the stabilization of the liquid of the bath, an amplitude value of temperature oscillation was defined for each sensor. For both temperature sensors, the amplitude values were lower than $0.05^{\circ} \mathrm{C}$. The temperature measurements of the samples varied within the amplitude value $22 \mathrm{~s}$ after the beginning of the heating of the spherical sensor. Figure 2 shows the temperature monitoring during a measurement cycle.

\section{Method validation}

The water content of the binary samples (ethanol and water mixtures) was compared to the reference measurements performed at the Organic Analysis Laboratory of Inmetro by means of a Karl Fischer coulometric titration. Table 1 presents for each sample the nominal ethanol content $E C_{N}$, the nominal water content $W C_{N}$, the reference water content $W C_{R}$ and their expanded uncertainties, with a coverage factor of $95.45 \%$.

The thermal conductivity of the samples was compared to literature values, since the institute does not have reference standards for measuring this quantity in liquids. A mean value was used as reference, once different values for the thermal conductivity of the samples at $20^{\circ} \mathrm{C}$ were found in the literatures.

Using a hot-wire equipment, Assael et al. [32] measured the thermal conductivity of the liquid mixtures of waterethanol, water-methanol and water-propanol in the range from $26.85^{\circ} \mathrm{C}$ to $71.85^{\circ} \mathrm{C}$. The thermal conductivity measurements were adjusted as a function of temperature, and the coefficients $\phi$ have been determined for pure components and for the mixtures, as shown in Table 2. The researchers compared their thermal conductivity values to five other works at $16.85^{\circ} \mathrm{C}$ and $56.85^{\circ} \mathrm{C}$ for the liquid mixtures of water-methanol and water-ethanol. The maximum difference between their measurements and those of the five works were $15 \%$, although this difference could be reduced to $5 \%$ when threes works were considered

$$
k=\phi_{1}+\phi_{2} T .
$$

Filippov [33] studied the thermal conductivity of liquid solutions as a function of their concentration. He analyzed twelve systems of unassociated components, ten systems with a single associated component, aqueous solutions of three alcohols and solutions of some salts and acids. From the thermal conductivities of the pure substances, an equation was proposed for estimating the thermal conductivity of binary mixtures:

$$
k=k_{1} w_{1}+k_{2} w_{2}-\lambda w_{1} w_{2}\left|k_{2}-k_{1}\right|
$$

where $k_{1}$ and $k_{2}$ are the thermal conductivities of the pure substances 1 and $2 ; w_{1}$ and $w_{2}$ are the mass fractions of these substances; and $\lambda$ is a coefficient that depends on the substances of the solution.

For solutions of unassociated substances, $\lambda$ is equal to 0.72 ; for solutions of associated substances, $\lambda$ ranges from 0.5 to 1 ; and for aqueous solutions it ranges from 0.3 to 0.7 . 
Table 3. Thermal conductivity values of the samples from literatures $\left(\right.$ in $\mathrm{W} / \mathrm{m}{ }^{\circ} \mathrm{C}$ ) at $20^{\circ} \mathrm{C}$.

\begin{tabular}{|c|c|c|c|c|c|}
\hline & \multicolumn{5}{|c|}{$W C_{N}(\%$ water $)$} \\
\hline & 0 & 25 & 50 & 75 & 100 \\
\hline Assael et al. [32] - Eq. (5) & 0.162 & 0.227 & 0.308 & 0.425 & 0.603 \\
\hline Fang et al. [41] & 0.164 & 0.229 & 0.315 & 0.445 & 0.598 \\
\hline Filippov [33] - Eq. (6) & - & 0.230 & 0.322 & 0.445 & - \\
\hline Reid [34] - Eq. (7) & - & 0.234 & 0.321 & 0.439 & - \\
\hline Melinder [42] & - & - & 0.317 & 0.438 & 0.598 \\
\hline KDB [37] - Eqs. (11) and (12) & 0.169 & - & - & - & 0.609 \\
\hline IAPWS [38] & - & - & - & - & 0.598 \\
\hline Ramires et al. [35] - Eq. (8) & - & - & - & - & 0.598 \\
\hline Vargaftik [43] & 0.168 & - & - & - & - \\
\hline Miller and Yaws [36] - Eq. (9) & 0.169 & - & - & - & - \\
\hline Touloukian et al. [34] - Eq. (10) & 0.168 & - & - & - & - \\
\hline Mean & 0.1666 & 0.2300 & 0.3169 & 0.4384 & 0.6006 \\
\hline Standard deviation of the mean & 0.0013 & 0.0015 & 0.0025 & 0.0037 & 0.0019 \\
\hline
\end{tabular}

According to Khan [34], based on 120 data, the value of $\lambda$ for Filippov's equation for liquid mixtures of water-ethanol in the range from $-70^{\circ} \mathrm{C}$ to $60^{\circ} \mathrm{C}$ is 0.571066 .

Reid et al. have also proposed an equation for estimating the thermal conductivity of binary mixtures as a function of the concentrations and thermal conductivities of the pure substances [34]:

$$
k=\left(k_{1} w_{1}^{\lambda}+k_{2} w_{2}^{\lambda}\right)^{1 / \lambda} .
$$

According to Khan [34], the value of $\lambda$ for the equation of Reid et al. for liquid mixtures of water-ethanol in the range from $-70^{\circ} \mathrm{C}$ to $60^{\circ} \mathrm{C}$ is 0.051892 .

From several experimental measurements performed by the hot-wire method, Ramires et al. [35] proposed an equation for estimating the thermal conductivity of water in the range from $0.85^{\circ} \mathrm{C}$ to $96.85^{\circ} \mathrm{C}$ at atmospheric pressure:

$$
\begin{aligned}
k & =0.6065 \\
& \times\left[-1.48445+4.12292\left(\frac{T}{298.15}\right)-1.63866\left(\frac{T}{298.15}\right)^{2}\right] .
\end{aligned}
$$

Miller and Yaws proposed an equation for estimating the thermal conductivity of ethanol in the range from $-114{ }^{\circ} \mathrm{C}$ to $190^{\circ} \mathrm{C}[36]$ :

$$
k=0.26293-3.8468 \times 10^{-4} T+2.2106 \times 10^{-7} T^{2} .
$$

Touloukian et al. proposed an equation for estimating the thermal conductivity of pure liquids, where for ethanol in the range from $-123{ }^{\circ} \mathrm{C}$ to $127^{\circ} \mathrm{C}$ the coefficients $\lambda_{1}$ and $\lambda_{2}$ are 609.512 and -0.70924 , respectively [34]:

$$
k=\left(\lambda_{1}+\lambda_{2} T\right) \times 0.0004187 .
$$

The Korea Thermophysical Properties Data Bank (KDB), which provides information and estimating methods for the thermophysical properties of several substances, presents the following equations for estimating the thermal conductivities of water (in the range from $-0.15^{\circ} \mathrm{C}$ to $349.85^{\circ} \mathrm{C}$ ) and ethanol (in the range from $-113.15^{\circ} \mathrm{C}$ to $\left.189.85^{\circ} \mathrm{C}\right)$, respectively [37]:

$$
\begin{aligned}
& k=-0.3838+0.005254 T-6.369 \times 10^{-6} T^{2}, \\
& k=-0.2629-0.0003847 T+2.211 \times 10^{-7} T^{2} .
\end{aligned}
$$

The International Association for the Properties of Water and Steam (IAPWS), a non-profit association of national organizations concerned with the properties of water and steam, provides in its website an online calculator, prepared by Moscow Power Engineering Institute and Russian National Committee of IAPWS, for estimating the thermal conductivity of water as a function of temperature and density [38]. The calculator was based on the "Release on the IAPWS Formulation 2011 for the Thermal Conductivity of Ordinary Water Substance" [39]. The density of water was estimated by the equation presented by Tanaka et al. [40]. The output value of thermal conductivity is presented in Table 3.

For estimating the thermal conductivity values of the binary samples by means of the equations (6) and (7), the thermal conductivities of the pure substances were necessary. In these cases, the thermal conductivities of ethanol and water were obtained, respectively, from KDB [37] and IAPWS [38]. Besides the thermal conductivity 
values estimated by the aforementioned equations for water, ethanol and their mixtures, Table 3 also presents values which were interpolated or directly obtained in tables available in literatures [41-43].

\section{Preliminary results}

For each sample, three measurement cycles were performed, and for each cycle a temperature step was determined as:

$$
\Delta T=\bar{T}^{f}-\bar{T}^{i}
$$

where, $\Delta T$ is the temperature step; $\bar{T}^{i}$ is the mean temperature of the five readings before the heating of the sphere; and $\bar{T}^{f}$ is the last five readings during the heating of sphere.

As explained before, during the calibration of the thermometers it was observed that the measurements varied within the range of $0.05^{\circ} \mathrm{C}$ in a stabilized medium. This parameter was adopted as criterion to determine the steady-state condition, which was reached $22 \mathrm{~s}$ after the heating of the sphere. The temperature step could then be determined in approximately $30 \mathrm{~s}$. However, a slightly longer measuring time was used.

Knowing the radius of the sphere, the heat transfer rate (electrical power) and the temperature step (measured as detailed in Sect. 3), the thermal conductivities of the samples could then be determined by means of equation (2). Nevertheless, the estimated results were highly unsatisfactory, since they were very diverging from the literature values shown in Table 3. The percentage differences from the estimated values to the literature ones increased from approximately 10-130\% (sensor 01) and $30-160 \%$ (sensor 02) as the ethanol content increased.

Another option could be to use an effective radius instead of the geometrical radius of the sphere. An effective radius, which can be estimated by the calibration of the spherical sensor in certain substances (selected by the user according to his measurement needs), consists of a virtual radius that compensates the lack of knowledge of some parameters involved in the measurement process, so as to accurately reproduce the thermal conductivities of the substances taken as reference. Using the thermal conductivities of the samples as reference (Tab. 3), the heat transfer rate and the temperature steps, the effective radii for each sensor could be estimated. Nevertheless, for both sensors, the effective radii varied considerably from one sample to another. The effective radius of the sensors increased linearly as the ethanol content increased. The estimated effective radii for sensors 01 and 02 were respectively $1.28 \mathrm{~mm}$ and $1.34 \mathrm{~mm}$ for distilled water, and $2.67 \mathrm{~mm}$ and $2.73 \mathrm{~mm}$ for anhydrous ethanol. Due to these considerable variations, the results were not useful for the measurement of the ethanol and water mixtures, because it would be necessary to use an effective radius value according to the concentration of the sample to be measured, which is usually unknown information in practice. Except for the half and half sample, the use of a mean effective radius is not convenient either. The percentage differences from the estimated thermal conductivities to the literature values increased as the difference of the concentrations of the solutions increased, reaching approximately $35 \%$ in the pure substances (distilled water and anhydrous ethanol).

The determination of the thermal conductivity values of the samples by the previous methods did not succeed, because equation (2) should only be used in ideal models, i.e., free from error sources. Nevertheless, the real process presents errors. So, a new model needed to be developed.

\section{Development of an experimental model}

In an ideal model, the temperatures of the sphere and the medium are the same in the interface. However, as shown in [25], the temperature measured in the sphere is shifted due to the thermal gradient within the sphere and the thermal contact resistance between the heated sphere and the surrounding medium. It can be stated as:

$$
T_{s}=T_{m}+\delta_{T G}+\delta_{T R}=T_{m}+\delta_{T},
$$

where $\delta_{T G}$ is the temperature shift due to the thermal gradient within the sphere; $\delta_{T R}$ is the temperature shift due to the thermal contact resistance between the sphere and the medium; and $\delta_{T}$ is the temperature shift due to $\delta_{T G}$ and $\delta_{T R}$.

Rearranging equation (14) as $\delta_{T}=T_{s}-T_{m}$, and using equations (2) and (4), $\delta_{T}$ can be deduced as:

$$
\delta_{T}=\frac{\dot{Q}}{4 \pi r_{s}}\left[\frac{1}{2 k_{s}}-\frac{r^{2}}{2 k_{s} r_{s}^{2}}+\frac{1}{r_{s} H_{c}}\right] .
$$

Rearranging equation (14) as $T_{m}=T_{s}-\delta_{T}$, equation (2) can be rewritten as:

$$
k=\frac{\dot{Q}}{4 \pi r_{s}\left(T_{s}-\delta_{T}\right)} .
$$

Two parameters were experimentally measured for the determination of the thermal conductivities of the samples: the heat transfer rate $\dot{Q}$ and the temperature step $\Delta T$, which means that $k$ should be expressed as a function of them. Equation (16) is valid when the initial temperature is zero; for the cases where it is not zero, $\Delta T$ is used instead of $T_{s}$. So, equation (16) can be rearranged as:

$$
k=\frac{\dot{Q}}{\Delta T} \times\left[\frac{1}{4 \pi r_{s}\left(1-\frac{\delta_{T}}{\Delta T}\right)}\right] .
$$

Equation (17) shows that the thermal conductivity of the medium can be determined by means of the product of the experimental ratio $\dot{Q} / \Delta T$ and a term, named as $\xi$, that takes into consideration the possible temperature errors. The term $\xi$ was calculated for each sample through the calibration of the spherical sensors by means of the 
Table 4. Experimental ratios $\dot{Q} / \Delta T$ and $\xi$ for each sample obtained at $20^{\circ} \mathrm{C}$.

\begin{tabular}{|c|c|c|c|c|c|}
\hline \multirow[t]{3}{*}{$W C_{N}(\%$ water $)$} & \multirow{3}{*}{$\begin{array}{l}k_{L} \\
\mathrm{~W} / \mathrm{m}^{\circ} \mathrm{C}\end{array}$} & \multicolumn{2}{|l|}{ Sensor 01} & \multicolumn{2}{|l|}{ Sensor 02} \\
\hline & & $\dot{Q} / \Delta T$ & $\xi$ & $\dot{Q} / \Delta T$ & $\xi$ \\
\hline & & $\mathrm{W} /{ }^{\circ} \mathrm{C}$ & $\mathrm{m}^{-1}$ & $\mathrm{~W} /{ }^{\circ} \mathrm{C}$ & $\mathrm{m}^{-1}$ \\
\hline 0 & 0.1666 & $5.598 \times 10^{-3}$ & 29.8 & $5.715 \times 10^{-3}$ & 29.2 \\
\hline 25 & 0.2300 & $6.433 \times 10^{-3}$ & 35.8 & $6.692 \times 10^{-3}$ & 34.4 \\
\hline 50 & 0.3169 & $7.556 \times 10^{-3}$ & 41.9 & $7.704 \times 10^{-3}$ & 41.1 \\
\hline 75 & 0.4384 & $8.859 \times 10^{-3}$ & 49.5 & $8.754 \times 10^{-3}$ & 50.1 \\
\hline 100 & 0.6006 & $9.680 \times 10^{-3}$ & 62.0 & $10.102 \times 10^{-3}$ & 59.4 \\
\hline
\end{tabular}

Table 5. Thermal conductivities of the samples by the developed model (in $\mathrm{W} / \mathrm{m}^{\circ} \mathrm{C}$ ) at $20^{\circ} \mathrm{C}$.

\begin{tabular}{|c|c|c|c|c|c|c|c|c|c|c|}
\hline \multirow[t]{3}{*}{ Sensor } & \multicolumn{10}{|c|}{$W C_{N}$ (\% water $)$} \\
\hline & \multicolumn{2}{|c|}{100} & \multicolumn{2}{|c|}{75} & \multicolumn{2}{|c|}{50} & \multicolumn{2}{|c|}{25} & \multicolumn{2}{|c|}{0} \\
\hline & $k$ & $\Delta \%$ & $k$ & $\Delta \%$ & $k$ & $\Delta \%$ & $k$ & $\Delta \%$ & $k$ & $\Delta \%$ \\
\hline \multirow[t]{3}{*}{01} & 0.598 & -0.4 & 0.441 & 0.6 & 0.318 & 0.3 & 0.230 & 0.0 & 0.165 & -1.0 \\
\hline & 0.600 & -0.1 & 0.437 & -0.3 & 0.317 & 0.0 & 0.231 & 0.4 & 0.166 & -0.4 \\
\hline & 0.603 & 0.4 & 0.437 & -0.3 & 0.317 & 0.0 & 0.230 & 0.0 & 0.170 & 2.0 \\
\hline \multirow[t]{3}{*}{02} & 0.601 & 0.1 & 0.434 & -1.0 & 0.314 & -0.9 & 0.225 & -2.2 & 0.166 & -0.4 \\
\hline & 0.603 & 0.4 & 0.440 & 0.4 & 0.319 & 0.7 & 0.230 & 0.0 & 0.167 & 0.2 \\
\hline & 0.597 & -0.6 & 0.439 & 0.1 & 0.321 & 1.3 & 0.232 & 0.9 & 0.167 & 0.2 \\
\hline
\end{tabular}

following equation:

$$
\xi=\frac{k_{L}}{\dot{Q} / \Delta T}
$$

where $k_{L}$ is the mean thermal conductivity of the sample obtained from literature.

Table 4 presents for each sample the experimental ratio $\dot{Q} / \Delta T$, which is a mean value of the three measurement cycles, $k_{L}$ and the terms $\xi$.

An equation relating the experimental ratios $\dot{Q} / \Delta T$ and the terms $\xi$ could be adjusted for each sensor. This equation is the calibration curve of the instrument. It means that, knowing $\dot{Q}$ and measuring $\Delta T$, the equation compensates the errors of the sensor and gives the thermal conductivity value for water, ethanol and any unknown mixture between these substances. Nevertheless, as the thermal conductivities of the binary samples do not vary linearly with the water content, a polynomial function is the best option. Thus, a third degree equation (with coefficient of determination equals 1) was then chosen for each sensor:

$$
\begin{aligned}
\text { Sensor } 01: & -1.877 \times 10^{-7} \xi^{3}+2.341 \times 10^{-5} \xi^{2} \\
- & 7.873 \times 10^{-4} \xi \\
+ & \left(1.324 \times 10^{-2}-\frac{Q}{\Delta T}\right) \\
= & 0
\end{aligned}
$$

$$
\begin{aligned}
\text { Sensor } 02: & 1.118 \times 10^{-7} \xi^{3}-1.561 \times 10^{-5} \xi^{2} \\
+ & 8.441 \times 10^{-4} \xi \\
& -\left(8.403 \times 10^{-3}-\frac{Q}{\Delta T}\right) \\
= & 0
\end{aligned}
$$

Table 5 presents the thermal conductivities of the samples, determined by means of the equations (19) and (20), for the three measurement cycles, and the percentage differences $\Delta \%$ from the estimated values to the literature ones.

Unlike the previous cases, the determination of the thermal conductivity values of the samples by the developed model was successful. The thermal conductivities estimated for the measured samples clearly present small divergences from the literature values, which show that the equations set for the sensors by means of calibration are fairly good.

The developed experimental model worked because the errors of the real measurement process were compensated by the calibration. As shown by equation (15), some errors of the measurement process come from the lack of some information, such as the thermal conductivity of the sphere, the right positioning of the temperature sensor within the sphere and the thermal resistance between the sphere and the medium. In addition, other phenomena may also cause errors in the process, such as the influence of the 
Table 6. Water content of the binary mixtures of water and ethanol at $20^{\circ} \mathrm{C}$.

\begin{tabular}{|c|c|c|c|c|c|c|c|c|}
\hline \multirow[t]{3}{*}{ Sensor } & \multicolumn{8}{|c|}{$W C_{N}(\%$ water $)$} \\
\hline & \multicolumn{2}{|c|}{5} & \multicolumn{2}{|c|}{25} & \multicolumn{2}{|c|}{50} & \multicolumn{2}{|c|}{75} \\
\hline & $\begin{array}{l}k \\
\mathrm{~W} / \mathrm{m}^{\circ} \mathrm{C}\end{array}$ & $\begin{array}{l}W C \\
\% \text { water }\end{array}$ & $\begin{array}{l}k \\
\mathrm{~W} / \mathrm{m}^{\circ} \mathrm{C}\end{array}$ & $\begin{array}{l}W C \\
\% \text { water }\end{array}$ & $\begin{array}{l}k \\
\mathrm{~W} / \mathrm{m}^{\circ} \mathrm{C}\end{array}$ & $\begin{array}{l}W C \\
\% \text { water }\end{array}$ & $\begin{array}{l}k \\
\mathrm{~W} / \mathrm{m}^{\circ} \mathrm{C}\end{array}$ & $\begin{array}{l}W C \\
\% \text { water } \\
\end{array}$ \\
\hline \multirow[t]{3}{*}{01} & 0.186 & 8.2 & 0.230 & 24.6 & 0.318 & 50.5 & 0.441 & 75.3 \\
\hline & 0.186 & 8.3 & 0.231 & 24.9 & 0.317 & 50.2 & 0.437 & 74.7 \\
\hline & 0.185 & 7.8 & 0.230 & 24.6 & 0.317 & 50.2 & 0.437 & 74.6 \\
\hline \multirow[t]{3}{*}{02} & 0.174 & 3.2 & 0.225 & 23.1 & 0.314 & 49.5 & 0.434 & 74.2 \\
\hline & 0.176 & 3.9 & 0.230 & 24.9 & 0.319 & 50.7 & 0.440 & 75.1 \\
\hline & 0.175 & 3.9 & 0.232 & 25.6 & 0.321 & 51.2 & 0.439 & 75.0 \\
\hline
\end{tabular}

sensor cable and the possibility of some convective effects. That is why it is very important to calibrate each heated sphere sensor individually, so that a compensation for the unknown errors may be included in the ideal model.

The determination of the water content $W C$ of the binary samples was done by means of the thermal conductivity measurements. Using the literature values (Tab. 3), a regression equation (a polynomial of third degree) was fitted correlating the water content to the thermal conductivity:

$$
\begin{aligned}
W C & =719.6059 k^{3}-1144.9714 k^{2}+757.1352 k \\
& -97.5994 .
\end{aligned}
$$

Due to the measurement uncertainty of the water content, the measurement of alcohol containing low concentrations of water, such as anhydrous ethanol (about $0.7 \%$ water) is not suitable. Thus, a sample of ethyl alcohol (about $5 \%$ water) was used. Table 6 presents for the three measurement cycles the thermal conductivity and the water content estimated for the binary mixtures of water and ethanol.

\section{Uncertainty analysis}

Based on the Guide to the Expression of Uncertainty in Measurement (GUM) [44], the combined standard uncertainties of the values of thermal conductivity and water content of the samples were respectively estimated as:

$u(k)=\sqrt{u^{2}\left(r_{k}\right)+u^{2}\left(s_{k}\right)+\left[c_{i}(k) u\left(f_{k}\right)\right]^{2}+\left[c_{i}(k) u\left(\frac{\dot{Q}}{\Delta T}\right)\right]^{2}}$,

where

$-u(k)$ is the combined standard uncertainty of $k$;

$-u(r)$ is the standard uncertainty due to the thermal conductivity resolution adopted for the equipment (based on a rectangular distribution);
- $u\left(s_{k}\right)$ is the standard uncertainty of the mean thermal conductivity of the sample obtained from literatures (based on a normal distribution);

- $u\left(f_{k}\right)$ is the standard uncertainty related to the polynomial equations (19) and (20) (based on a normal distribution);

$-u(\dot{Q} / \Delta T)$ is the standard uncertainty related to the experimental ratio $\dot{Q} / \Delta T$ (based on a normal distribution), which combined the uncertainties of the individual components $\dot{Q}$ and $\Delta T$;

$-c_{i}(k)$ is the coefficient sensitivity of the thermal conductivity to a particular input, estimated as the first order derivate of $k$ with respect to the experimental ratio $\dot{Q} / \Delta T$.

$$
u(W C)=\sqrt{u^{2}\left(r_{W C}\right)+u^{2}\left(f_{W C}\right)+\left[c_{i}(W C) u(k)\right]^{2}},
$$

where

$-u(W C)$ is the combined standard uncertainty of $W C$;

$-u\left(r_{W C}\right)$ is the standard uncertainty due to the water content resolution adopted for the equipment (based on a rectangular distribution;

$-u\left(f_{W C}\right)$ is the standard uncertainty related to the polynomial equation (21) (based on a normal distribution);

$-u(k)$ is the combined standard uncertainty of $k$;

$-c_{i}(W C)$ is the coefficient sensitivity of the water content to a particular input, estimated as the first order derivate of $W C$ with respect to $k$.

The expanded uncertainties of the values of thermal conductivity and water content of the samples were respectively estimated as:

$$
U(k)=\kappa \times u(k),
$$

$$
U(W C)=\kappa \times u(W C),
$$

where $U(k)$ is the expanded uncertainty of the thermal conductivity; $\kappa$ is the coverage factor ( $\kappa$ equals two was used, which gives a coverage probability of $95.45 \%$ ); and $U$ $(W C)$ is the expanded uncertainty of the water content. 
Table 7. Uncertainties for the thermal conductivities of the samples.

\begin{tabular}{|c|c|c|c|c|c|c|c|c|}
\hline Sensor & $W C_{N}(\%$ water $)$ & $\begin{array}{l}u\left(r_{k}\right) \\
\mathrm{W} / \mathrm{m}^{\circ} \mathrm{C}\end{array}$ & $\begin{array}{l}u\left(s_{k}\right) \\
\mathrm{W} / \mathrm{m}{ }^{\circ} \mathrm{C}\end{array}$ & $\begin{array}{l}c_{i}(k) u\left(f_{k}\right) \\
\mathrm{W} / \mathrm{m}^{\circ} \mathrm{C}\end{array}$ & $\begin{array}{l}c_{i}(k) u(\dot{Q} / \Delta T) \\
\mathrm{W} / \mathrm{m}^{\circ} \mathrm{C}\end{array}$ & $\begin{array}{l}u(k) \\
\mathrm{W} / \mathrm{m}^{\circ} \mathrm{C}\end{array}$ & $\begin{array}{l}U(k) \\
\mathrm{W} / \mathrm{m}{ }^{\circ} \mathrm{C}\end{array}$ & $\begin{array}{l}U(k) \\
\%\end{array}$ \\
\hline \multirow[t]{5}{*}{01} & 0 & 0.000289 & 0.0013 & 0.0007 & 0.0032 & 0.0035 & 0.007 & 4.2 \\
\hline & 25 & 0.000289 & 0.0015 & 0.0006 & 0.0053 & 0.0056 & 0.011 & 4.8 \\
\hline & 50 & 0.000289 & 0.0025 & 0.0007 & 0.0074 & 0.0079 & 0.016 & 5.0 \\
\hline & 75 & 0.000289 & 0.0037 & 0.0009 & 0.0087 & 0.0095 & 0.019 & 4.3 \\
\hline & 100 & 0.000289 & 0.0019 & 0.0011 & 0.0138 & 0.0139 & 0.028 & 4.7 \\
\hline \multirow[t]{5}{*}{02} & 0 & 0.000289 & 0.0013 & 0.0014 & 0.0047 & 0.0050 & 0.010 & 6.0 \\
\hline & 25 & 0.000289 & 0.0015 & 0.0019 & 0.0055 & 0.0060 & 0.012 & 5.2 \\
\hline & 50 & 0.000289 & 0.0025 & 0.0025 & 0.0088 & 0.0095 & 0.019 & 6.0 \\
\hline & 75 & 0.000289 & 0.0037 & 0.0030 & 0.0132 & 0.0140 & 0.028 & 6.4 \\
\hline & 100 & 0.000289 & 0.0019 & 0.0029 & 0.0178 & 0.0181 & 0.036 & 6.0 \\
\hline
\end{tabular}

Table 8. Uncertainties for the water content of the binary mixtures.

\begin{tabular}{lllllll}
\hline Sensor & $W C_{N}(\%$ water $)$ & $\begin{array}{l}u\left(r_{W C}\right) \\
\text { \% water }\end{array}$ & $\begin{array}{l}u\left(f_{W C}\right) \\
\text { \% water }\end{array}$ & $\begin{array}{l}c_{i}(W C) u(k) \\
\% \text { water }\end{array}$ & $\begin{array}{l}u(W C) \\
\% \text { water }\end{array}$ & $\begin{array}{l}U(W C) \\
\% \text { water }\end{array}$ \\
\hline 01 & 5 & 0.029 & 0.390 & 1.583 & 1.63 & 3.3 \\
& 25 & 0.029 & 0.390 & 1.891 & 1.93 & 3.9 \\
& 50 & 0.029 & 0.390 & 1.980 & 2.02 & 4.1 \\
& 75 & 0.029 & 0.390 & 1.586 & 1.63 & 3.3 \\
02 & & & & & \\
& 5 & 0.029 & 0.390 & 2.324 & 2.36 & 4.8 \\
& 25 & 0.029 & 0.390 & 2.029 & 2.07 & 4.2 \\
& 50 & 0.029 & 0.390 & 2.315 & 2.35 & 4.7 \\
& 75 & 0.029 & 0.390 & 2.493 & 2.52 & 5.1 \\
\hline
\end{tabular}

It is worth pointing out that the reproducibility of $\Delta T$ is due to the reproducibility of the temperature measurements, which was estimated during the calibration of the thermometer (by means of the repetition of the calibration point $20^{\circ} \mathrm{C}$ ) and included in its uncertainty. The repeatability of $\Delta T$ was estimated during the temperature measurements of the samples as the standard deviation of the mean values (before and during the heating of the sphere). In the case of $\dot{Q}$, the reproducibility consists on how the equipment reproduces the heat generation. It was estimated during the characterization of the equipment (by means of the repetition of three calibration points) and included in its uncertainty. The repeatability of $\dot{Q}$ was also estimated during the characterization of the equipment by means of the standard deviation of the mean values of voltage and current and included in its uncertainty.

Table 7 presents the standard uncertainties, the combined standard uncertainty, the expanded uncertainty and the percentage expanded uncertainty of the thermal conductivity determined for each sample.

Table 8 presents the standard uncertainties, the combined standard uncertainty and the expanded uncertainty of the water content determined for each sample.

\section{Comparing the results}

The thermal conductivity values reported in some literatures were not provided with their uncertainty statements or error estimations. So, the agreement of the measured values with the literature ones was checked by means of the percentage expanded uncertainties of the thermal conductivities determined for the samples. The percentage differences between all the values of thermal conductivity given in the literatures (by means of tables or by the equations from (6) to (12)) and the mean thermal conductivity measured for each sample (calculated by means of the values of the three measurement cycles) were smaller than its percentage expanded uncertainty, which confirm the agreement of the measured values with the literature ones. Figures 3 (sensor 01) and 4 (sensor 02) show the difference between each literature value and the mean thermal conductivity, and the uncertainty of the thermal conductivity measurement of each sample.

It can be clearly seen by means of Figures 3 and 4 that there is a good agreement between the measured thermal conductivities and the literature ones for all the samples, which validates the developed method for measuring the thermal conductivity of water, ethanol and their mixtures. 


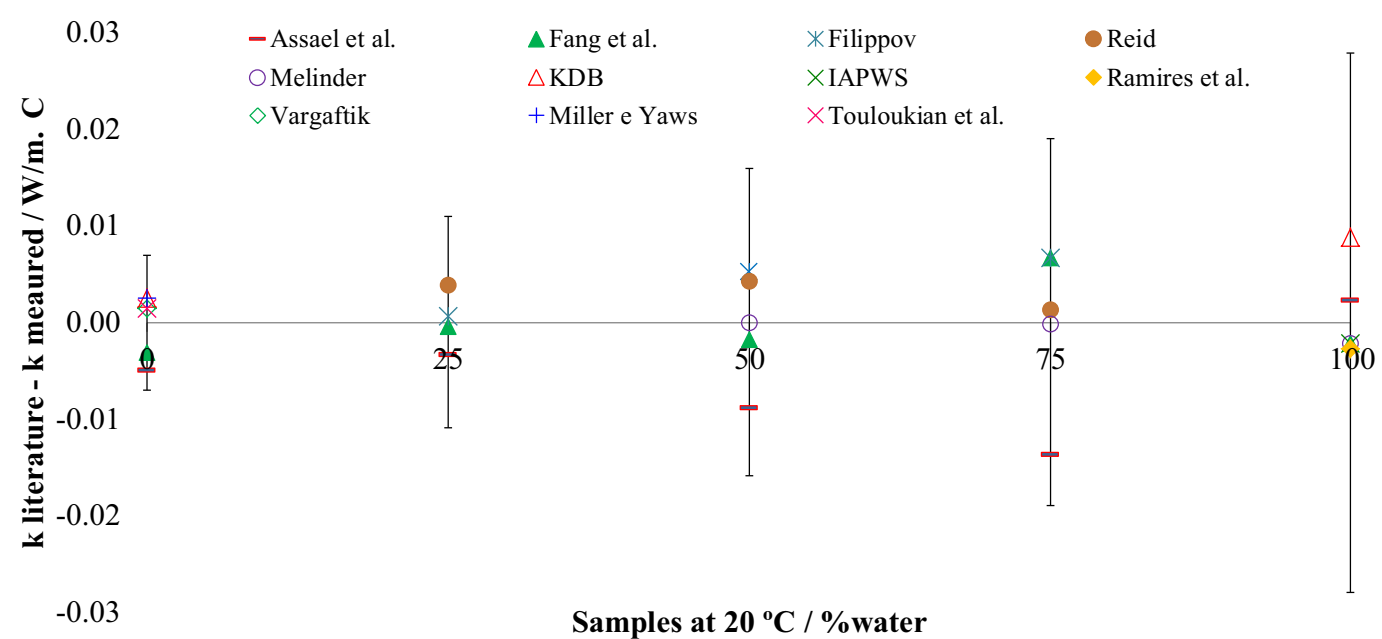

Fig. 3. Differences between literature values and the mean thermal conductivity for sensor 01 .

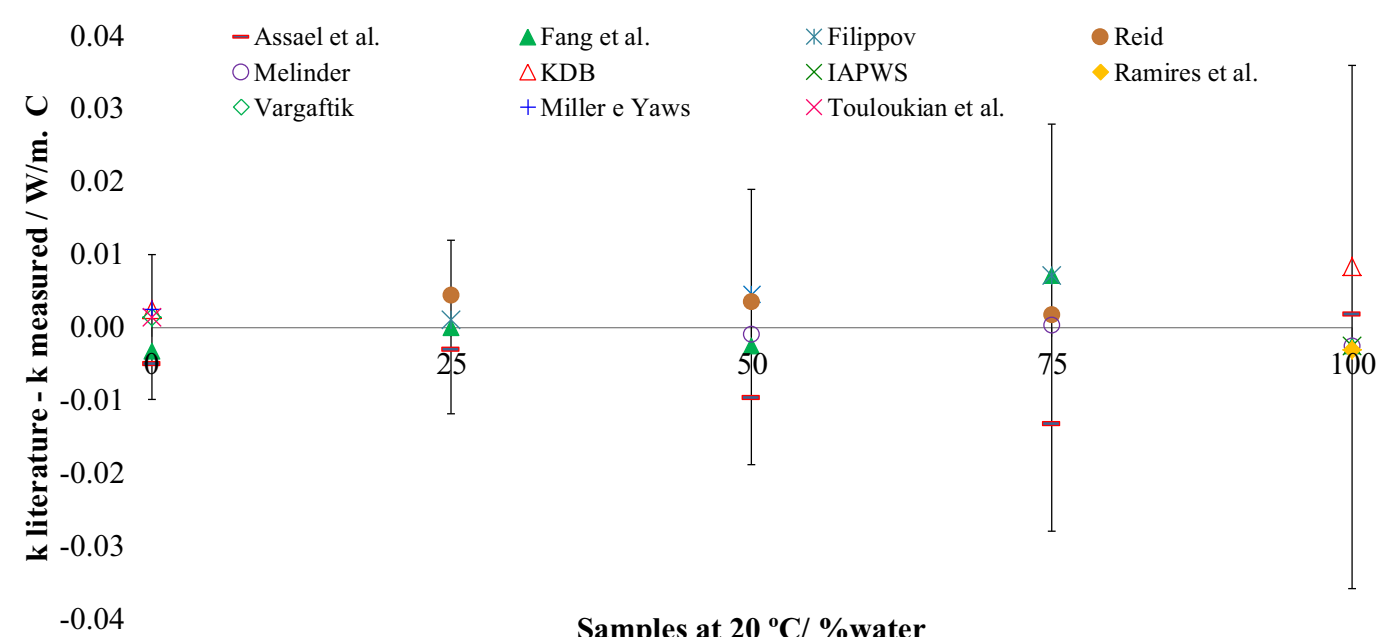

Fig. 4. Differences between literature values and the mean thermal conductivity for sensor 02 .

Table 9. Comparison of the measurements of $W C$ of the binary samples (in \% water).

\begin{tabular}{|c|c|c|c|c|c|c|c|c|}
\hline \multirow[t]{2}{*}{$W C_{N}$} & \multirow[t]{2}{*}{$W C_{R}$} & \multirow[t]{2}{*}{$U\left(W C_{R}\right)$} & \multicolumn{3}{|c|}{ Sensor 01} & \multicolumn{3}{|c|}{ Sensor 02} \\
\hline & & & $W C$ & $U(W C)$ & $E_{n}$ & $W C$ & $U(W C)$ & $E_{n}$ \\
\hline \multirow[t]{3}{*}{5} & 5.368 & 0.001 & 8.2 & 3.3 & 0.8 & 3.2 & 4.7 & 0.5 \\
\hline & & & 8.3 & 3.3 & 0.9 & 3.9 & 4.7 & 0.3 \\
\hline & & & 7.8 & 3.3 & 0.7 & 3.9 & 4.7 & 0.3 \\
\hline \multirow[t]{3}{*}{25} & 25.5270 & 0.2143 & 24.6 & 3.9 & 0.2 & 23.1 & 4.1 & 0.6 \\
\hline & & & 24.9 & 3.9 & 0.2 & 24.9 & 4.1 & 0.2 \\
\hline & & & 24.6 & 3.9 & 0.2 & 25.6 & 4.1 & 0.0 \\
\hline \multirow[t]{3}{*}{50} & 50.3596 & 0.2862 & 50.5 & 4.0 & 0.0 & 49.5 & 4.7 & 0.2 \\
\hline & & & 50.2 & 4.0 & 0.0 & 50.7 & 4.7 & 0.1 \\
\hline & & & 50.2 & 4.0 & 0.0 & 51.2 & 4.7 & 0.2 \\
\hline \multirow[t]{3}{*}{75} & 75.1930 & 0.1509 & 75.3 & 3.3 & 0.0 & 74.2 & 5.0 & 0.2 \\
\hline & & & 74.7 & 3.3 & 0.1 & 75.1 & 5.0 & 0.0 \\
\hline & & & 74.6 & 3.3 & 0.2 & 75.0 & 5.0 & 0.0 \\
\hline
\end{tabular}


In order to validate the water content determined for the binary samples, these were compared to the reference measurements performed at Inmetro and presented in Table 1 . The compatibility of the measurements was checked by means of the normalized error $\left(E_{n}\right)$, which is calculated according to the following equation [45]:

$$
E_{n}=\frac{W C-W C_{R}}{\sqrt{U(W C)^{2}+U\left(W C_{R}\right)^{2}}} .
$$

Table 9 shows the reference measurements of the water content of the binary samples, the values determined by the developed method in this study and the normalized errors.

A comparison between two measurements is satisfactory when $\left|E_{n}\right| \leq 1$. Consequently, the water content measurements of this work and those performed by the reference laboratory are clearly compatible, since all the $E_{n}$ values were lower than one, validating the developed method for measuring the water content of binary samples of water and ethanol.

\section{Conclusions}

The method of the spherical heat source, in principle, is an absolute measuring method of thermal conductivity, which means that the sensor can provide an output without being calibrated against a standard or a reference material. However, some parameters of the model need to be carefully taken into consideration. Thus, to compensate for the lack of some theoretical evaluations and the difficulty for obtaining accurately some practical information, the heated sphere sensors needed to be calibrated by means of mediums with known thermal properties. As the devices have different constructive characteristics from each other, the calibration must be done individually.

The use of spherical heat sources in the industrial sectors for measuring the thermal conductivity presents considerable advantages, such as wide measuring range, relatively fast measurements, measurement uncertainty compatible with other techniques and the possibility of using the sensor in situ. The method has also a great potential to be employed in research institutes and laboratories that provide calibration and testing services.

The applicability of the method of the spherical heat source for measuring the thermal conductivity of water, ethanol and their mixtures proved to be quite satisfactory, since the measurements of this study showed excellent agreement with the values proposed by several researchers. This agreement occurred with values obtained by other measurement methods, such as the traditional hot-wire technique, as with those obtained by means of equations for estimating the thermal conductivity. The applicability of the method for determining the water content of the binary samples was also quite satisfactory, since the results of the proposed method showed good agreement with those performed by the reference equipment from the national institute (Inmetro).

\section{Implications and influences}

The paper presents the metrological aspects and the calibration procedure of a spherical heat source to measure thermal conductivity and water content of some liquid samples. This will strongly contribute and stimulate future works on the investigation of the applicability of the spherical heat source method and its metrological aspects for measuring thermal conductivity and water content of other liquids and other mediums.

\section{References}

1. M.J. Assael, K.D. Antoniadis, W.A. Wakeham, Int. J. Thermophys. 31, 1051 (2010)

2. ASTM D-2717-15, Standard Test for Thermal Conductivity of Liquids (American Society for Testing and Materials, Philadelphia, PA, 2009)

3. U. Hammerschmidt, W. Sabuga, Int. J. Thermophys. 21, 1255 (2000)

4. F.A. Gibbs, Proc. Soc. Exp. Biol. Med. 31, 141 (1933)

5. J. Grayson, J. Physiol. 114 (Suppl.), 29 (1951)

6. W. Chester, J. Grayson, Nature 4273, 521 (1951)

7. J. Grayson, J. Physiol. 118, 54 (1952)

8. H.S. Carslaw, in Introduction to the Mathematical Theory of the Conduction of Heat in Solids (Macmillan, London, 1921), 2nd ed.

9. J.C. Chato, Therm. Prob. Biotechnol. Trans. ASME 16 (1968)

10. T.A. Balasubramaniam, H.F. Bowman, J. Heat Trans. Trans. ASME 296 (1974)

11. R.K. Jain, J. Biomech. Eng. Trans. ASME 101, 82 (1979)

12. M.M. Chen, K.R. Holmes, V. Rupinskas, J. Biomech. Eng. Trans. ASME 103, 253 (1981)

13. J.W. Valvano, The use of thermal diffusivity to quantify tissue perfusion, Ph.D. thesis, Harvard University, 1981

14. G. Hamilton, Investigation of the thermal properties of human and animal tissues, Ph.D. thesis, University of Glasgow, 1998

15. H. Zhang, S. Cheng, L. He, A. Zhang, Y. Zheng, D. Gao, Cell Preserv. Technol. 1, 141 (2002)

16. K.A. Woodbury, Experimental and analytical investigation of liquid moisture distribution in roof insulating systems, Ph.D. thesis, Virginia Polytechnic Institute and State University, 1984

17. M. Fujii, H. Takamatsu, T. Fujii, in Proceedings of the 1st Asian Thermophysical Properties Conference, Beijing, 1986, edited by W. Buxuan et al. (China Academic Publishers, Beijing, 1986), pp. 462-467

18. B.P. Dougherty, An automated probe for thermal conductivity measurements, M.Sc. thesis, Virginia Polytechnic Institute and State University, 1987, cited in [22]

19. R.R. Kravets, Determination of thermal conductivity of food materials using a bead thermistor, Ph.D. thesis, Virginia Polytechnic Institute and State University, 1988, cited in [22]

20. U.B. Holeschovsky, G.T. Martin, J.W. Tester, Int. J. Heat Mass Transf. 39, 1135 (1996)

21. S. Radhakrishnan, Measurement of thermal properties of seafood, M.Sc. thesis, Virginia Polytechnic Institute and State University, 1997 
22. M.F. Gelder, A thermistor based method for the measurement of thermal conductivity and thermal diffusivity of moist food materials at high temperatures, $\mathrm{Ph} . \mathrm{D}$. thesis, Virginia Polytechnic Institute and State University, 1998

23. H. Zhang, L. He, S. Cheng, Z. Zhai, D. Gao, Meas. Sci. Technol. 14, 1396 (2003)

24. L. Kubicar, L. Bagel, V. Vretenar, V. Stofanik, in Proceedings of the Meeting of the Thermophysical Society - Thermophysics 2005, Kocovce, 2005, edited by P. Matiasovsky, O. Koronthalyova (ICA SAS, Bratislava, 2005), pp. 38-42

25. L. Kubicar, V. Vretenar, V. Stofanik, V. Bohac, Int. J. Thermophys. (2008), doi:10.1007/s10765-008-0544-4

26. L. Kubicar, U. Hammerschmidt, D. Fridrikova, P. Dieska, V. Vretenar, in Proceedings of Thermophysics 2010, Valtice, 2010 (Brno University of Technology, Brno, 2010), pp. 166-171

27. J. Goldemberg, L.A.H. Nogueira, http://bioenergyconnec tion.org/article/sweetening-biofuel-sector-history-sugar cane-ethanol-brazil, accessed on May 2016

28. H.S. Carslaw, J.C. Jaeger, in Conduction of Heat in Solids (Oxford University Press, London, 1959), 2nd ed.

29. H.F. Bowman, T.A. Balasubramaniam, Cryobiology 13, 572 (1976)

30. J.W. Valvano, J.R. Cochran, K.R. Diller, Int. J. Thermophys. 6, 301 (1985)

31. R.J. Schmidt, S.W. Milverton, Proc. Roy. Soc. Lond. A: Mater. 152, 586 (1935)

32. M.J. Assael, E. Charitidou, W.A. Wakeham, Int. J. Thermophys. 10, 793 (1989)

33. L.P. Filippov, Int. J. Heat Mass Transf. 11, 331 (1968)

34. M.H. Khan, Modeling, simulation and optimization of ground source heat pump systems, M.Sc. thesis, Oklahoma State University, 2004
35. M.L.V. Ramires, C.A.N. de Castro, Y. Nagasaka, A. Nagashima, M.J. Assael, W.A. Wakeham, J. Phys. Chem. Ref. Data 24, 1377 (1995)

36. S. Henke, P. Kadlec, Z. Bubník, J. Food Eng. 99, 497 (2010)

37. Korea Thermophysical Properties Data Bank, KDB: Pure Components Properties (2012), available on http://www. cheric.org, accessed on 2012

38. IAPWS, Online Calculation for General and Scientific Use (2012), available on http://www.iapws.org/relguide/ ThCond.html, accessed on 2012

39. IAPWS, Release on the IAPWS Formulation 2011 for the Thermal Conductivity of Ordinary Water Substance (2012), available on http://www.iapws.org/release.html, accessed on 2012

40. M. Tanaka, G. Girard, R. Davis, A. Peuto, N. Bignell, Metrologia 38, 301 (2001)

41. L.Q. Fang, L.R. Sen, N.D. Yan, H.Y. Chun, J. Chem. Eng. Data 42, 971 (1997)

42. A. Melinder, Thermophysical properties of aqueous solutions used as secondary working fluids, Ph.D. thesis, Royal Institute of Technology, 2007

43. B.E. Polin, J.M. Prausnitz, J.P. O'Connell, in The Properties of Gases and Liquids (McGraw-Hill, New York, 2001), 5th ed.

44. INMETRO, Avaliação de Dados de Medição: Guia para a Expressão da Incerteza de Medição - GUM2008 (INMETRO, Rio de Janeiro, 2012), 1st ed.

45. Conformity Assessment - General Requirements for Proficiency Testing, ISO/IEC 17043 (International Organization for Standardization and International Electrotechnical Commission, 2010)

Cite this article as: Júlio Dutra Brionizio, Alcir de Faro Orlando, Georges Bonnier, Characterization of a spherical heat source for measuring thermal conductivity and water content of ethanol and water mixtures, Int. J. Metrol. Qual. Eng. 8, 18 (2017) 